\title{
06.2
}

\section{Флексоэлектрические домены в бинарной смеси нематиков}

\author{
() В.А. Делев, Ю.И. Тимиров \\ Институт фризики молекул и кристаллов - обособленное структурное подразделение Федерального государственного \\ бюджетного научного учреждения Уфимского федерального исследовательского центра РАН, Уфа, Россия \\ E-mail: delev@anrb.ru
}

Поступило в Редакцию 21 мая 2020 г.

В окончательной редакции 30 сентября 2020 r.

Принято к публикации 6 октября 2020 г.

Исследовано образование флексоэлектрических доменов в смеси нематических жидких кристаллов $\mathrm{N}$-(4-Methoxybenzylidene)-4-butylaniline и 4-Cуano-4'-pentylbiphenyl с анизотропией диэлектрической проницаемости $\varepsilon_{a} \approx 0$ под действием постоянного напряжения. Измерены пороговые характеристики доменной структуры (пороговое напряжение и пространственный период) в исследуемой смеси. Получена оценка разности флексоэлектрических коэффициентов $\left|e_{1}-e_{3}\right|$. Показано, что в смеси нематических жидких кристаллов с $\varepsilon_{a} \approx 0$ пороговое напряжение определяется исключительно отношением $k /\left(e_{1}-e_{3}\right)$, где $k-$ средняя константа упругости. Это позволяет вычислить разность флексокоэффициентов $\left|e_{1}-e_{3}\right|$ напрямую, исключая свойства поверхностей. Предложенный и апробированный метод определения флексоэлектрических коэффициентов может быть полезным для новых синтезированных нематических жидких кристаллов с $\varepsilon_{a}<-0.5$.

Ключевые слова: нематический жидкий кристалл, флексоэлектрическая неустойчивость, флексоэлектрические домены, флексоэлектрические коэффициенты.

DOI: 10.21883/PJTF.2021.02.50542.18388

Существует целый ряд явлений, которые благодаря сочетанию ориентационного порядка с подвижностью молекул наблюдаются только в жидких кристаллах [1-4]. Одним из них является флексоэлектрический эффект, суть которого состоит в возникновении поляризации при деформации средней преимущественной ориентации молекул жидких кристаллов (директора $\mathbf{n}$ ). При этом деформация поля директора в однородном электрическом поле инициируется границами ячейки, и конечная энергия сцепления директора с поверхностью служит необходимым условием ее возникновения в одномерном случае. Таким образом, для наблюдения флексодеформации и определения флексокоэффициентов энергия сцепления директора с поверхностью играет важную роль [4]. Однако в смеси нематических жидких кристаллов (НЖК, нематик) с $\varepsilon_{a} \approx 0$ пороговое напряжение определяется исключительно отношением $k /\left(e_{1}-e_{3}\right)$ (здесь $k-$ средняя константа упругости НЖК), что позволяет вычислить разность флексокоэффициентов $\left|e_{1}-e_{3}\right|$ напрямую, исключая свойства поверхностей [4].

В ряде нематиков при приложении к планарному слою постоянного напряжения выше некоторого порогового значения $U_{c}$ возникает флексоэлектрическая неустойчивость, наблюдаемая в виде продольных доменов, ориентированных вдоль исходного направления директора $\mathbf{n}$. Согласно теории [5-8], образование флексодоменов в планарном слое НЖК возможно только в определенной ограниченной области значений материальных параметров НЖК, отвечающих за неустойчивость, таких как разность флексокоэффициентов $\left|e_{1}-e_{3}\right|$, анизотропия диэлектрической проницаемости $\varepsilon_{a}=\varepsilon_{\|}-\varepsilon_{\perp} \quad\left(\varepsilon_{\|}-\right.$ направление вдоль директора, $\varepsilon_{\perp}-$ перпендикулярно директору), константы упругости $k_{11}$ и $k_{22}$ для деформаций поперечного изгиба и кручения (твист-деформация). Этим, в частности, объясняется тот факт, что до сих пор флексодомены не наблюдались в планарных слоях чистого нематика N-(4-Methoxybenzylidene)-4-butylaniline (MBBA) с $\varepsilon_{a}=-0.53$, так как при $\varepsilon_{a} \approx-0.5$ порог неустойчивости стремится к бесконечности: $U_{c} \rightarrow \infty$ [9].

К настоящему времени разработан ряд различных методов измерения флексоэлектрических коэффициентов, как правило, для случая гибридной ориентации НЖК (когда директор ориентирован планарно на одной из подложек и гомеотропно на другой). Однако результаты для суммы $\left|e_{1}+e_{3}\right|$ и/или разности $\left|e_{1}-e_{3}\right|$ флексокоэффициентов, в частности, для МВВА, полученные различными методами, зачастую значительно расходятся не только по величине, но и по знаку.

Такой разброс значений затрудняет, например, количественный теоретический анализ влияния флексоэффекта на формирование электроконвективных структур в НЖК, где его роль, как показано в [10-13], может быть весьма существенной.

С другой стороны, флексоэлектрический эффект может использоваться в устройствах отображения информации, для создания управляемых электрическим полем дифракционных решеток, а также цветных индикаторных устройств [14].

Таким образом, в настоящее время проблема определения флексоэлектрических коэффициентов НЖК остается актуальной не только с фундаментальной точки зрения, но и в плане технических приложений. 

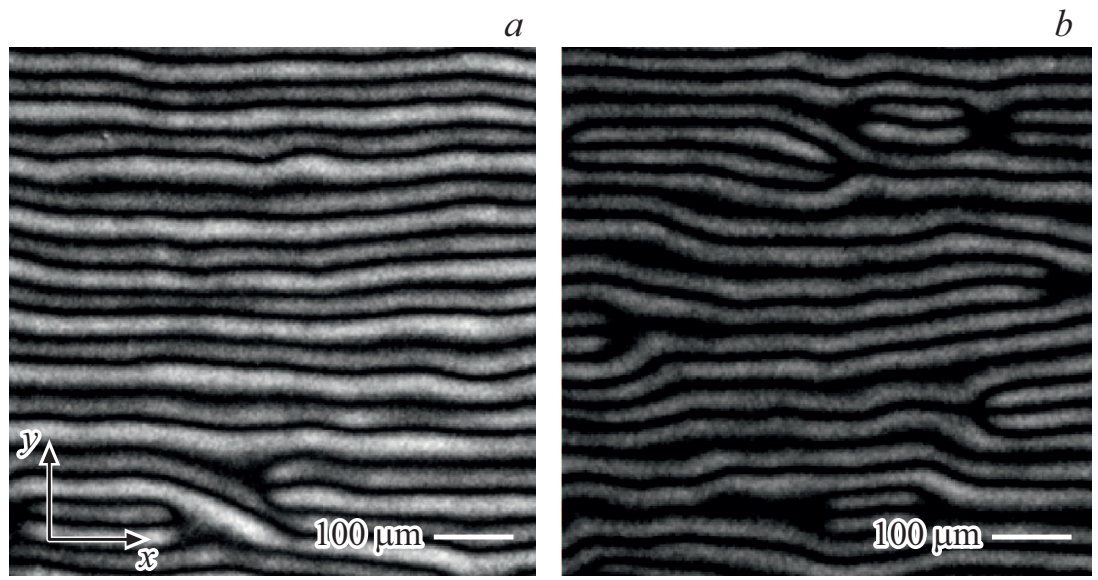

$b$

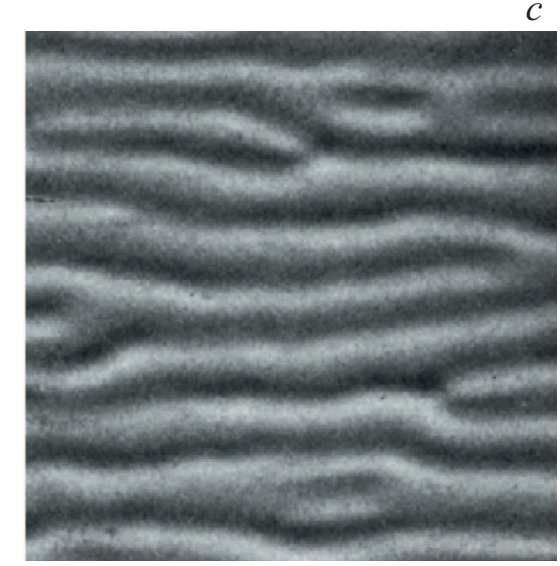

Рис. 1. Флексодомены в смеси НЖК с $\varepsilon_{a} \approx 0 . a-U=3.25 \mathrm{~V}, \Lambda=85 \mu \mathrm{m} ; b-U=4.6 \mathrm{~V}, \Lambda=70 \mu \mathrm{m} ; c-U=4.6 \mathrm{~V}$ (с фазовой пластинкой $\lambda / 4)$. Поляризатор и анализатор скрещены, при этом поляризатор перпендикулярен директору. Толщина ЖК-ячейки $d=40 \mu \mathrm{m}$.

Целью настоящей работы является изучение флексоэффекта в бинарной смеси нематиков МBВА и 4-Cyano-4'-pentylbiphenyl (5CB) с диэлектрической анизотропией $\varepsilon_{a} \approx 0$. Поскольку в смеси НЖК с компенсированной диэлектрической анизотропией $\varepsilon_{a} \approx 0$ пороговое напряжение определяется исключительно соотношением $k /\left(e_{1}-e_{3}\right)$, можно вычислить флексоэлектрические коэффициенты напрямую, исключая свойства поверхностей [4]. Для этого исходя из зависимости контраста изображений доменных структур от приложенного к слою НЖК постоянного напряжения необходимо определить порог образования флексодоменов и затем получить оценку разности флексокоэффициентов в рамках теории Бобылева-Пикина [5].

Для получения бинарной смеси НЖК с $\varepsilon_{a} \approx 0$ использовались жидкие кристаллы МВВА и 5СВ с диэлектрической анизотропией $\varepsilon_{a}^{\mathrm{MBBA}}=-0.53$ и $\varepsilon_{a}^{5 \mathrm{CB}}=+13.3$ при $25^{\circ} \mathrm{C}[15,16]$. Диэлектрическая анизотропия смеси определялась по стандартной формуле

$$
\varepsilon_{a}=w t \varepsilon_{a}^{\mathrm{MBBA}}+(1-w t) \varepsilon_{a}^{5 \mathrm{CB}},
$$

где $w t=0.96-$ весовая доля МВВА в смеси с $\varepsilon_{a} \approx 0$. После приготовления смесь НЖК с $\varepsilon_{a} \approx 0$ заключалась между двумя прозрачными подложками с проводящим покрытием из $\mathrm{SnO}_{2}$, разделенными майларовыми прокладками заданной толщины. Планарная ориентация создавалась натиранием поверхностей электродов в одном направлении. Для изучения зависимости флексоэффекта от толщины жидкокристаллического (ЖК) слоя использовались прокладки толщиной $d=13,23$ и $40 \mu \mathrm{m}$. Все измерения проводились при комнатной температуре $\left(T=25^{\circ} \mathrm{C}\right)$.

Возникающие под действием приложенного постоянного напряжения пространственно-периодические структуры наблюдались в поляризационно-оптический микроскоп Zeiss Axio Imager, a их изображения регистрировались при помощи CCD-камеры PCO VX44 с разрешением $512 \times 512$ точек, частотой кадров $25 \mathrm{~Hz}$ и 256 уровнями серого цвета. Оцифрованные изображения обрабатывались на компьютере.

Для определения порогов образования пространственно-периодических структур приложенное напряжение изменялось от $U=0 \mathrm{~V}$ до $U>U_{c}$ с шагом $\Delta U=50 \mathrm{mV}$. ЖК-образец выдерживался в течение 5 min при данном напряжении, чтобы обеспечить релаксацию возможных переходных процессов (характерное время релаксации составляет $\sim 1-10 \mathrm{~s})$. Контраст полученных изображений $C$ вычислялся по формуле $[17,18]$ :

$$
C=\left\langle\sqrt{\frac{1}{512}} \sum_{i=1}^{512}\left(\frac{I_{i j}}{I_{j}^{a v}}-1\right)^{2}\right\rangle,
$$

где $I_{i j}$ - интенсивность $i$-го пиксела в $j$-й линии, $I_{j}^{a v}-$ средняя величина интенсивности $j$-й линии, а угловые скобки означают усреднение по различным линиям. Для определения контраста каждого изображения использовалось, как правило, 100 линий, ориентированных перпендикулярно направлению ориентации доменной структуры. Линейный фитинг зависимости контраста $C$ от приложенного напряжения $U$ вблизи порога позволяет достаточно точно определить критическое напряжение $U_{c}$ образования пространственно-периодической структуры. Пространственный период $\Lambda$ структур определялся с помощью двумерного фурье-преобразования оцифрованных изображений. Возможность возникновения гидродинамических потоков в пространственнопериодических структурах контролировалась с помощью анализа движения пробных частиц диаметром 2-4 $\mu \mathrm{m}$, добавленных в НЖК. Основные результаты изложены для ячейки с толщиной слоя НЖК $d=40 \mu \mathrm{m}$. Заметим, что в этом случае регистрация гидродинамических потоков в ЖК-образце методом пробных частиц может быть проведена более точно, чем в случае ячеек меньшей толщины. 

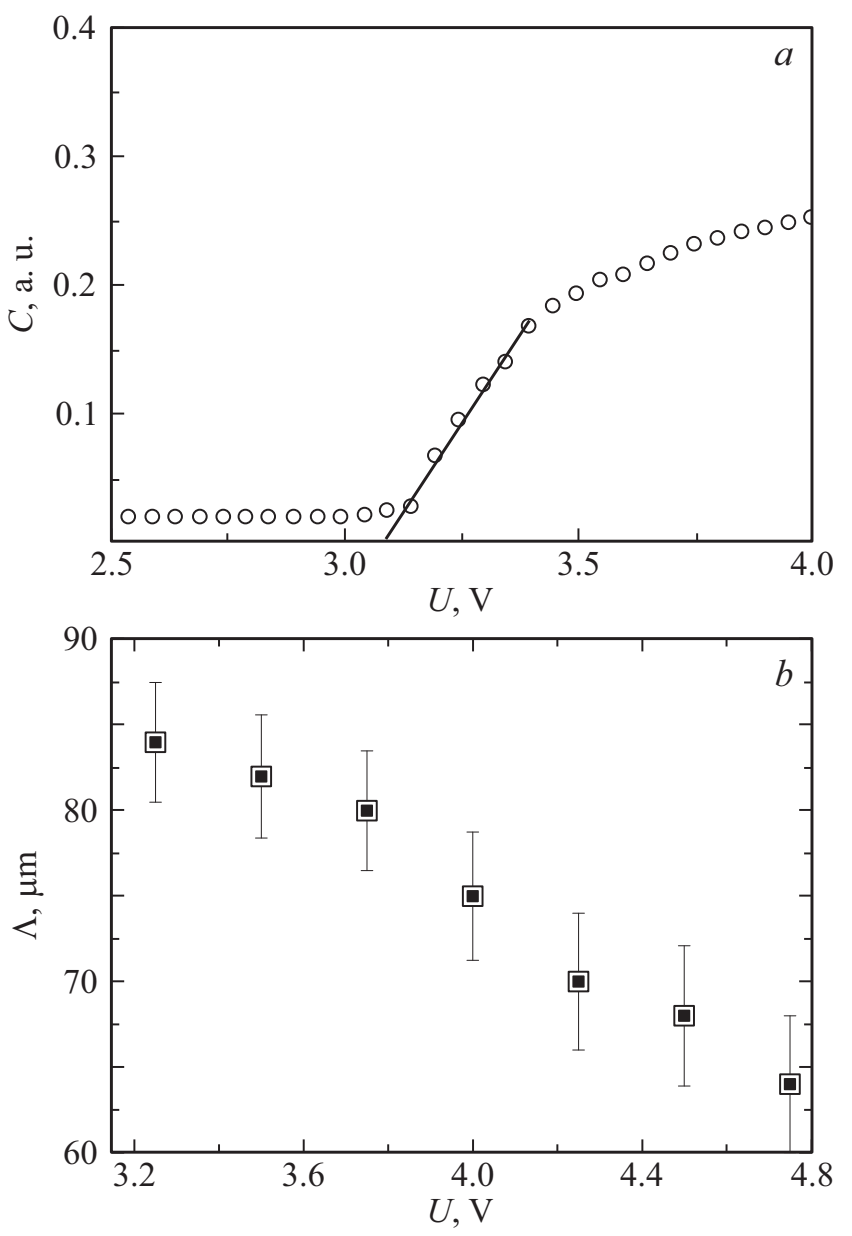

Рис. 2. $a-$ зависимость контраста $C$ от приложенного напряжения $U ; b-$ зависимость пространственного периода флексодоменов $\Lambda$ от приложенного напряжения $U$.

Рассмотрим структурные превращения, возникающие в смеси НЖК под действием постоянного приложенного напряжения. На рис. 1 представлены изображения флексодоменов, которые ориентированы вдоль исходной планарной ориентации директора $\mathbf{n} \| \mathbf{x}$ на подложках ЖК-ячейки и являются периодическими вдоль оси $\mathbf{y}$ На рис. 1, а изображение флексодоменов представлено вблизи порога $U \sim U_{c}$. На рис. $1, b$ изображены домены, полученные при более высоком напряжении. Установлено, что с увеличением приложенного напряжения пространственный период доменной структуры уменьшается, при этом растет число дефектов (рис. $1, b)$.

Для ЖК-ячеек различной толщины $(d=13,23$ и $40 \mu \mathrm{m})$ пороговые напряжения образования флексоэлектрических доменов практически не изменяются и составляют $U_{c}=3.2 \pm 0.1 \mathrm{~V}$ (рис. $2, a$ ). Пространственный период (ширина) доменной структуры $\Lambda$ при $U=U_{c}$ растет линейно с увеличением толщины слоя смеси НЖК $\Lambda_{c} / d=2.13 \pm 0.1$ и уменьшается с увеличением приложенного напряжения выше порога (рис. 2, b). При смене полярности пороги не меняются в отличие от случая ЖК-ячеек с гибридной ориентацией [19].

Поляризационно-оптический анализ с использованием фазовой пластинки $\lambda / 4$ для усиления вклада твист-моды $\pm \varphi$ в контраст полученного изображения доменной структуры показал (рис. $1, c$ ), что образование флексодоменов сопровождается выходом директора из плоскости $(x z)$ исходной планарной ориентации на угол $\pm \varphi$ в соседних полупериодах флексоэлектрических доменов.

При увеличении приложенного напряжения $U \geqslant 5.0 \mathrm{~V}$ наблюдалось вращение пробных частиц, что свидетельствует о возникновении электроконвективной моды. С дальнейшим увеличением приложенного напряжения электроконвективная мода подавляет флексоэлектрическую неустойчивость.

Проведем оценку разности флексоэлектрических коэффициентов $\left|e_{1}-e_{3}\right|$ исследуемой смеси НЖК, используя формулу Пикина [5]; с учетом того, что $\varepsilon_{a}=0$, получим

$$
\left|e_{1}-e_{3}\right|=2 \pi k / U_{c},
$$

где $k=\left(k_{11}+k_{22}\right) / 2=5.43 \cdot 10^{-12} \mathrm{~N}-$ средняя константа упругости НЖК. Подставляя $U_{c}=3.2 \mathrm{~V}$, получим $\left|e_{1}-e_{3}\right| \approx 1.07 \cdot 10^{-11} \mathrm{C} / \mathrm{m}$, что близко к оценке флексокоэффициентов НЖК МВВА $\left|e_{1}-e_{3}\right| \approx 1.70 \cdot 10^{-11} \mathrm{C} / \mathrm{m}$, полученной полуэмпирическим методом [18,19], а также к величине $\left|e_{1}-e_{3}\right| \approx 1.40 \cdot 10^{-11} \mathrm{C} / \mathrm{m}$, определенной в [20]. Для периода флексодоменов имеем, согласно теории Бобылева-Пикина, $\Lambda_{c} / d \approx 2$, в нашем случае с учетом погрешностей измерений $\Lambda_{c} / d=2.13 \pm 0.1$.

Таким образом, в работе, по-видимому, впервые наблюдались флексодомены в бинарной смеси НЖК MBВА и 5СВ. Определена разность флексокоэффициентов $\left|e_{1}-e_{3}\right|$ смеси НЖК с $\varepsilon_{a}=0$. При оценке величины $\left|e_{1}-e_{3}\right|$ основная задача для бинарной смеси НЖК с $\varepsilon_{a}=0$ сводится к достаточно точному экспериментальному измерению весовых долей компонентов смеси и определению порога возникновения флексодоменов $U_{c}$. Предложенный в работе метод может являться альтернативным для определения флексокоэффициентов новых синтезированных НЖК с $\varepsilon_{a}<-0.5$.

\section{Благодарности}

Авторы благодарны А.П. Крехову за полезные дискуссии.

\section{Финансирование работы}

Работа выполнена в рамках государственного задания № AAAA-A19-119022290052-9.

\section{Конфликт интересов}

Авторы заявляют, что у них нет конфликта интересов. 


\section{Список литературы}

[1] R.B. Meyer, Phys. Rev. Lett., 22 (18), 918 (1969). https://doi.org/10.1103/PhysRevLett.58.1538

[2] П. де Жен, Физика жсидких кристаллов (Мир, М., 1977).

[3] С.А. Пикин, Структурные превращения в жидких кристаллах (Наука, М., 1981).

[4] Л.М. Блинов, Жидкие кристаллы. Структура и свойства (ЛИБРОКОМ, М., 2013).

[5] Ю.П. Бобылев, С.А. Пикин, ЖЭТФ, 72 (1), 369 (1977).

[6] Y.P. Bobylev, V.G. Chigrinov, S.A. Pikin, J. de Phys. Coll., 40 (C3), 331 (1979). https://doi.org/10.1051.jphyscol.1979364

[7] Flexoelectricity in liquid crystals. Theory, experiments and applications, ed by A. Buka, N. Eber (Imperial College Press, 2012).

[8] A. Krekhov, W. Pesch, Á. Buka, Phys. Rev. E, 83 (5), 051706 (2011). https://doi.org/10.1103/PhysRevE.83.051706

[9] М.И. Барник, Л.М. Блинов, А.Н. Труфанов, Б.А. Уманский, ЖЭТФ, 73 (5), 1936 (1977).

[10] A. Krekhov, W. Pesch, N. Eber, T. Toth-Katona, A. Buka, Phys. Rev. E, 77 (2), 021705 (2008).

https://doi.org/10.1103/PhysRevE.77.021705

[11] T. Toth-Katona, N. Eber, A. Buka, Mol. Cryst. Liq. Cryst., 511 (1), 11/[1481] (2009).

https://doi.org/10.1080/15421400903048461

[12] Э.С. Батыршин, А.П. Крехов, О.А. Скалдин, В.А. Делев, ЖЭТФ, 141 (6), 1200 (2012). [Пер. версия: https://doi.org/10.1134/S1063776112040061].

[13] N. Eber, P. Salamon, A. Buka, Liq. Cryst. Rev., 4 (2), 101 (2016). https://doi.org/10.1080/21680396.2016.1244020

[14] D.J. Gardiner, S.M. Morris, F. Castles, M.M. Qasim, W.-S. Kim, S.S. Choi, H.-J. Park, I.-J. Chung, H.J. Coles, Appl. Phys. Lett., 98 (26), 263508 (2011). https://doi.org/10.1063/1.3605597

[15] H. Kneppe, F.Schneider, N.K. Sharma, J. Chem. Phys., 77 (6), 3203 (1982). https://doi.org/10.1063/1.444195

[16] M.J. Bradshaw, E.P. Raynes, J.D. Bunning, T.E. Faber, J. de Phys., 46 (9), 1513 (1985). https://doi.org/10.1051/jphys:019850046090151300

[17] В.А. Делев, О.А. Скалдин, Письма в ЖТФ, 30 (16), 36 (2004).

[18] В.А. Делев, А.П. Крехов, ЖЭТФ, 152 (6), 1414 (2017). [Пер. версия: https://doi.org/10.1134/S1063776117120032].

[19] S.P. Palto, N.J. Mottram, M.A.Osipov, Phys. Rev. E, 75 (6), 061707 (2007). https://doi.org/10.1103/PhysRevE.75.061707

[20] T. Takahashi, S. Hashidate, H. Nishijou, M. Usui, M. Kimura, T. Akahane, Jpn. J. Appl. Phys., 37 (4A), 1865 (1998). https://doi.org/10.1143/JJAP.37.1865 